\title{
Choosing a Hyperacute Stroke Imaging Protocol for Proper Patient Selection and Time Efficient Endovascular Treatment: Lessons from Recent Trials
}

\author{
Jin Soo Lee, ${ }^{a}$ Andrew M. Demchuk ${ }^{\mathrm{b}}$ \\ ${ }^{a}$ Department of Neurology, Ajou University School of Medicine, Ajou University Hospital, Suwon, Korea \\ ${ }^{\mathrm{b}}$ Calgary Stroke Program, Departments of Clinical Neurosciences and Radiology, Hotchkiss Brain Institute, University of Calgary, Calgary, AB, Canada
}

Recently, several prospective randomized control trials regarding endovascular treatment for patients with intracranial large artery occlusions causing acute ischemic stroke have been successfully reported. Effort to minimize time delays to endovascular treatment, patient selection and the use of retrievable stent were important factors for the success of these trials. The inclusion and exclusion criteria for each of these trials did include differences in imaging protocols. In this review, we focus on the importance of baseline non-invasive angiography prior to deciding endovascular treatment. Then imaging protocols are described for each trial according to measurement of infarct volume and collateral grading.

Keywords Cerebral infarction; Thrombectomy; Endovascular procedures; X-ray computed tomography; Magnetic resonance imaging
Correspondence: Andrew M. Demchuk Calgary Stroke Program, Departments of Clinical Neurosciences and Radiology, Hotchkiss Brain Institute, University of Calgary, Calgary, $A B$, Canada

Email: ademchuk@ucalgary.ca

Received: August 25, 2015

Revised: September 20, 2015

Accepted: September 21, 2015

The authors have no financial conflicts of interest.

\section{Introduction}

Since about 1995, intravenous recombinant tissue plasminogen activator has been a gold standard for patients with acute ischemic stroke. ${ }^{1-3}$ For a diagnosis of acute ischemic stroke, noncontrast computed tomography (CT) was the only essential test beyond a clinical assessment based on history of illness, timing of last seen normal and neurological examination to rule out mimics and determine if disabling deficits. ${ }^{1,2}$ Intravenous tissue plasminogen activator (tPA) has since been shown to have limited efficacy in the setting of proximal large artery occlusions. ${ }^{4,5}$ Unfortunately, endovascular treatment with first generation devices failed to prove its efficacy ${ }^{6-8}$ until the advent of newer thrombectomy devices known as stent retrieval devices which resulted in a series of successful randomized clinical trials all published in 2015. .13 $^{-13}$
Several factors influenced the success of these recent endovascular treatment (Table 1). Retrievable stents such as (Solitaire FR [Medtronic, Irvine, CA, USA] and Trevo [Stryker, Kalamazoo, MI, USA]) were a leap in technology that achieved much higher rates of successful reperfusion than the older technology such as Merci (Concentric Medical, Mountain View, CA, USA) or intra-arterial thrombolytic delivery. ${ }^{14,15}$ Trials also mandated time targets for imaging to groin puncture and imaging to reperfusion which forced trial centres to focus on unnecessary delays and improve efficiencies. Initial CT to intra-arterial puncture times less than 1 hour were achieved in some these trials. ${ }^{10,11} \mathrm{~Pa}$ tient selection insured that all subjects had proximal intracranial occlusions and small or medium sized infarct cores of irreversible injury at time of baseline imaging. This selection used either a noncontrast CT (Alberta Stroke Program Early CT score [ASPECTS] based), CT angiography (CTA) collaterals or CT per- 
Table 1. Comparison of key points between failed and successful clinical trials regarding endovascular treatment for acute ischemic stroke

\begin{tabular}{|c|c|c|c|c|c|c|c|}
\hline Study name & Large artery occlusion & Infarct core/perfusion & Collateral criteria & Onset to puncture & Stent retriever & $\mathrm{mTICI} 2 \mathrm{~b} / 3$ & Ordinal shift mRS* \\
\hline \multicolumn{8}{|l|}{ Previous failed clinical trials } \\
\hline IMS-3 $3^{6}$ & N.R. & N.R. & N.R. & $249 \min$ & $1.2 \%$ & $41 \%$ & N.S. \\
\hline SYNTHESIS Expansion ${ }^{7}$ & N.R. & N.R. & N.R. & $225 \mathrm{~min}$ & $12.7 \%$ & N.A. & N.S. \\
\hline MRRESCUE ${ }^{8}$ & CTA or MRA & Core $<90 \mathrm{cc}(>1.43)^{\dagger}$ & N.R. & $381 \mathrm{~min}$ & $0 \%$ & $27 \%$ & N.S. \\
\hline \multicolumn{8}{|c|}{ Recent successful clinical trials } \\
\hline MR CLEAN ${ }^{9}$ & CTA & N.R. & N.R. & $260 \mathrm{~min}$ & $82 \%$ & $59 \%$ & $1.7(1.2-2.3)$ \\
\hline ESCAPE $^{10}$ & CTA & $\begin{array}{l}\text { NCCT, CBV or } \\
\text { CBF ASPECTS } \geq 6\end{array}$ & Good/moderate & $185 \mathrm{~min}^{\ddagger}$ & $86 \%$ & $72 \%$ & $2.6(1.7-3.8)$ \\
\hline SWIFT PRIME ${ }^{11}$ & CTA or MRA & $\begin{array}{r}\text { Core }^{\S}<50 \mathrm{~mL}(>1.8)^{\dagger} \\
\text { NCCT ASPECTS } \geq 6\end{array}$ & N.R. & $184 \min ^{\ddagger}$ & $100 \%$ & $88 \%$ & $2.6(1.6-4.4)$ \\
\hline EXTEND IA ${ }^{12}$ & CTA or MRA & Core $^{\| \prime}<70 \mathrm{~mL}(>1.2)^{\dagger}$ & N.R. & $210 \mathrm{~min}$ & $100 \%$ & $86 \%$ & N.A. \\
\hline REVASCAT $^{13}$ & CTA or MRA & $\begin{array}{r}\text { NCCT ASPECTS } \geq 7 \\
\text { DWI ASPECTS } \geq 6\end{array}$ & N.R. & $269 \mathrm{~min}$ & $100 \%$ & $66 \%$ & $1.7(1.1-2.8)$ \\
\hline
\end{tabular}

*Common odds ratio; 'Target mismatch ratio; 'Sum of median of parameters; ${ }^{\circledR}$ The ischemic core was assessed by MRI or CT: "The ischemic core was defined by regional cerebral blood flow on CT perfusion or diffusion-weighted imaging.

mTICl, modified Thrombolysis in Cerebral Infarction; mRS, modified Rankin Scale; N.R., not required; N.S., not significant; N.A., not available; CTA, computed tomography angiography; NCCT, noncontrast computed tomomgraphy; CBV, cerebral blood volume; CBF, cerebral blood flow; MRA, magnetic resonance angiography.

fusion (CTP) approach to insure that large core subjects were excluded from randomization. Previous failed studies targeted patients with acute ischemic stroke based on noncontrast CT only which led to the inclusions of subjects without acute intracranial large artery occlusions. ${ }^{16,17}$ One challenge with interpreting the 5 endovascular randomized trials is the use of slightly different imaging protocols that were used to insure selection of small to medium infarct cores. ${ }^{18-22}$ What are the pros and cons of these different protocols to help a centre determine which is most appropriate for their setting?

In this review, we will evaluate these protocols by examining common components such as baseline non-invasive angiography and different components such as infarct volume and collateral grading.

\section{Baseline non-invasive angiography occlusion detection}

\section{Previous failed trials}

Among patients with acute ischemic stroke, the target for endovascular treatment are large artery proximal occlusions involving the carotid artery, middle cerebral artery, or basilar artery. This was not a pre-requisite for enrollment in the 3 major clinical trials published in 2013. ${ }^{6-8}$ This resulted in enrollment of nonoccluded subjects unlikely to derive any benefit from endovascular treatment. ${ }^{16,17}$ The IMS-3 and SYNTHESIS Expansion studies did not use baseline CTA or magnetic resonance angiography (MRA) but rather chose imaging criteria typically performed for intravenous $\mathrm{PA}$ decision making of just noncontrast $\mathrm{CT}^{6,7} \mathrm{In}$ the IMS-3 trial only 334/434 (77\%) subjects underwent endovascular treatment despite being randomized to the endovascu- lar arm because many had no or distal target occlusions that were not amenable to treatment (supplemental figure 1 in reference 6). ${ }^{6}$ The IMS-3 subpopulation that did undergo baseline CTA and had an intracranial large artery occlusions (LAO) did have a tendency toward better outcomes with endovascular treatment. ${ }^{23}$

\section{Recent successful trials}

Recent successful clinical trials appropriately targeted subjects with intracranial LAO involving the anterior circulation by either baseline CTA or MRA. In all trials a crucial inclusion criteria was the confirmation of intracranial LAO at baseline. ${ }^{18-22}$ In REVASCAT, this criteria was taken one step further by mandating a vascular imaging study only after intravenous tPA administration for 30 minutes. ${ }^{22}$ This was put in place to capture subjects refractory to intravenous tPA induced early recanalization. Similarly, in MR CLEAN, a vast majority of subjects received only noncontrast CT imaging at baseline at the initial hospital prior to intravenous tPA so as to not delay standard treatment. These subjects were then administered intravenous tPA if eligible and subsequently transported to an endovascular hospital. At the endovascular hospital they then had vascular imaging performed (usually 60-90 minutes after intravenous tPA) which explains the large time difference between intravenous tPA bolus and randomization seen in MR CLEAN. Both trials resulted in a control population that was "tPA refractory" in many cases resulting in low rates of good outcomes in the control arms. The major difficulty with this approach adopted by REVASCAT and MR CLEAN was the longer median times to reperfusion seen compared to other trials which performed baseline vascular imaging at the same time as initial noncontrast 
CT in most cases.

Among LAO locations, proximal M1 occlusions were very efficacious in both ESCAPE and SWIFT PRIME..$^{10,11,24}$ Whereas the involvement of the internal carotid artery was one of the most efficacious occlusion locations with endovascular treatment in several of the trials, a large effect size warrants further post-hoc analysis. Involvement of the cervical carotid artery was also associated with a dramatic benefit for endovascular treatment in ESCAPE (common odds ratio 9.6 [2.6-35.5]). ${ }^{10}$

\section{Baseline infarct core volume estimation}

\section{CT or MRI ASPECTS}

Endovascular revascularization treatment should be done when the extent of salvageable brain tissue is significant and the extent of irreversible injury still modest. On this ground, evaluation of small core detection has been used to select proper patients. A pragmatic approach to infarct core estimation is using a semiquantitative early ischemic change ASPECTS methodology. ${ }^{19}$ This can be easily done without any specific software using only noncontrast brain CT or diffusion-weighted imaging (DWI) sequence of magnetic resonance imaging (MRI). ${ }^{25}$ The major challenge of noncontrast CT ASPECTS is the low interobserver reliability reported by several groups. ${ }^{26,27}$ Early ischemic change detection by ASPECTS is least reliable when the noncontrast CT scan is performed very early from symptom onset ( $<90$ minutes) at a time when the Hounsfield Unit drop from ischemia is still modest and therefore barely perceptible to the human eye. ${ }^{28}$ ASPECTS has been shown to predict clinical outcome after intravenous or intra-arterial revascularization treatments in previous studies. ${ }^{29-31}$ An ASPECTS 6-10 or 7-10 selection was successful at demonstrating the benefit of endovascular treatment in three recent trials (ESCAPE, SWIFT PRIME, and REVASCAT).$^{9-11,13}$ The only recent endovascular trial that randomized subjects without an ASPECTS threshold was MR CLEAN. In this trial the subgroup analysis by baseline ASPECTS suggested no treatment effect in those subjects with ASPECTS 4 or less (OR 1.09 [0.14-8.46]). ${ }^{9}$

DWI ASPECTS with MRI may be more reliable and predictive of clinical outcome than noncontrast CT ASPECTS ${ }^{32}$ but was rarely performed in the five recent endovascular trials. A DWI ASPECTS was applied in both SWIFT PRIME and the REVASCAT study. A DWI ASPECTS score less than 6 was an exclusion criterion in both SWIFT PRIME and REVASCAT. REVASCAT had a similar tiered approach to an ASPECTS threshold determined by age. ASPECTS score less than 7 was an exclusion if age less than 81 and ASPECTS score less than 9 was an exclusion if age 81 and 85 . Given the predominance of
CT over MRI in the recent endovascular trials, the role of MRI and DWI as first imaging tool should remain limited to sites who can perform such studies while maintaining door to reperfusion times that are comparable to noncontrast CT/CTA as the first imaging approach.

\section{Infarct core volume}

Infarct core volume determination has emerged as one way to determine reperfusion treatment eligibility. This can be accomplished with DWI the easiest by automated imaging software or by semi-quantitative hand drawing. Controversy remains as to what apparent diffusion coefficient threshold should be used as core volume. An apparent diffusion coefficient threshold of $600 \times 10^{-6} \mathrm{~mm}^{2} / \mathrm{s}$ is generally used and does correlate well with clinical outcomes. ${ }^{33}$ The recent endovascular trials, however, rarely used DWI volumes for patient eligibility because of the strong emphasis in limiting delays best accomplished by a first imaging, noncontrast CT/CTA approach. Only the SWIFT PRIME trial enrolled by MRI criteria in a portion of cases $(n=33)$. CTP was the primary imaging tool used for measurement of infarct core in two of the endovascular trials with the assistance of an automated software tool entitled RAPID (iSchemaView, Menlo Park, CA, USA).$^{34}$ Among CTP parameters, the ischemic core was defined as regional cerebral flow less than $30 \%$ of that in normal tissue in the EXTEND-IA ${ }^{12}$ whereas regional cerebral blood volume or delayed time to the peak of the residual function was used to identify ischemic core in previous studies. ${ }^{35-37}$ Comparing the two trials that used core volume, SWIFT PRIME chose a lower cutoff of core volume to exclude patients ( $>50 \mathrm{~mL}$ ) while EXTENDIA chose a higher cutoff of $>70 \mathrm{~mL} \cdot{ }^{20,21}$ Both trials included mismatch criteria comparing core volume to a penumbra threshold. The importance of assessing mismatch versus core volume alone remains unclear from the trials. One could argue that all endovascular trial subjects with carotid or M1 occlusions are likely to have a penumbra that is much larger than the core if the core volume is small given the territory an internal carotid or proximal middle cerebral artery supplies ( $>250 \mathrm{~mL}$ in most cases). RAPID-aided core volume threshold in a proximal occlusion would seem the simplest way to determine endovascular eligibility. This approach seems to rival a simpler noncontrast CT ASPECTS approach. The major limitation of CTP as the selection tool of choice is the additional time and radiation exposure required by the test. CTP data is also frequently corrupted by patient motion limiting its generalizability. The speed of patient enrollment was much faster in the clinical trials that did not mandate CTP for patient eligibility reflecting the limitations of this approach. 


\section{Leptomeningeal collateral grading with single or multiphase CTA}

There is a large body of evidence to suggest that leptomeningeal collaterals play a very crucial role in maintaining blood flow to brain regions distal to an arterial occlusion. ${ }^{38}$ Imaging assessment of leptomeningeal collaterals in humans relies on an indirect assessment of the extent and rate of backfilling of pial arteries receiving blood flow through small inter-arteriolar connections. ${ }^{39-42}$ Significant intracerebral hemorrhage risk $(25.0 \%)$ has been seen in patients with poor pial collaterals especially when recanalization is achieved. ${ }^{43,44} \mathrm{~A}$ number of CTA-based collateral scoring systems have been published which correlate collateral extent with clinical outcome. Good collateral status as assessed by CTA was significantly associated with small initial infarct volume, reduced infarct expansion, and more favorable functional outcomes. ${ }^{39,45-49}$ CTA also has the added advantage of estimating collaterals backfilling for both anterior-middle cerebral artery and posterior-middle cerebral artery collaterals. This is not possible with selective carotid injections during angiography which do not provide information on collateral backfilling from the posterior circulation to middle cerebral artery via posterior cerebral artery. In the IMS-3 trial, baseline CTA collaterals were a robust determinant of final clinical outcome with maximal benefit for endovascular treatment seen in patients with intermediate collaterals. ${ }^{50}$ The ESCAPE trial was the first acute stroke trial to utilize CTA-based collateral assessment for patient selection. ESCAPE excluded patients with poor or no collaterals in greater than $50 \%$ of the middle cerebral artery territory. ${ }^{19}$ The trial allowed standard single phase CTA but encouraged use of multiphase CTA. Single phase CTA is a snapshot in time that can miss crucial pathophysiology such as delayed collateral backfilling ${ }^{51}$ that is best appreciated with imaging several seconds after peak arterial phase acquisition. Multiphase CTA generates time-resolved cerebral angiograms of brain vasculature from the skull base to the vertex in three phases after contrast injection by movement of the $\mathrm{CT}$ gantry over the cranium three times approximately 8 seconds apart. Multiphase CTA was slightly better in the prediction of clinical out- come than currently used techniques such as noncontrast $\mathrm{CT}$, single phase CTA, and CTP. ${ }^{52}$ In ESCAPE collateral assessment augmented noncontrast CT ASPECTS interpretation. Site investigators were urged to review noncontrast $\mathrm{CT}$ early ischemic changes using ASPECTS in combination with and in the context of the CTA collateral assessment. Large regions of early ischemic change (low ASPECTS) were taught to be questioned if collaterals appeared robust throughout the same regions. Further refinement of CTA collateral grading is needed for ease of use by clinicians.

\section{How to choose amongst several imaging protocols for endovascular treatment decision making}

\section{Noncontrast CT, single phase CTA (1 step, 1 contrast)}

Most institutions have their own unique imaging protocols to properly select patients with acute ischemic stroke for revascularization treatment (Table 2). The combination of noncontrast CT and CTA is the most pragmatic approach most frequently used in the recent endovascular trials. It can easily be performed in only a few minutes and provides sufficient information to determine suitability for endovascular treatment in most scenarios. The detection of an occlusion in an intracranial large artery is essential to endovascular treatment decision making. In the MR CLEAN study, this approach was all that was required and the study was successful. The noncontrast CT/CTA approach relies heavily on ASPECTS and collateral assessment to rule out subjects with large ischemic cores. An ASPECTS cutoff of 4 and below appears to identify the large core group. In MR CLEAN no evidence of treatment effect was seen with endovascular treatment in the ASPECTS 0-4 group. Further studies are needed, however, in this low ASPECTS group especially when ultraearly reperfusion achieved ( $<3$ hours from onset) before we conclude endovascular treatment is always futile in this low ASPECTS population. Collateral assessment using single phase CTA has limitations as it may underestimate the extent of collateral flow due to early imaging after bolus. Despite this limitation it certainly can complement noncontrast CT ASPECTS as

Table 2. Evaluations of several imaging protocols regarding patient selection for endovascular treatment

\begin{tabular}{|c|c|c|c|c|}
\hline Imaging approach & NCCT/sCTA & NCCT/mCTA & NCCT/CTA/CTP & $\mathrm{mMRl}$ \\
\hline Time efficiency (short door-to-reperfusion time) & +++ & +++ & $H$ & + \\
\hline Detection of large artery occlusions & +++ & $+H+$ & $+1+$ & ++ \\
\hline Ischemic core estimation & + & ++ & $+1+$ & +++ \\
\hline Technical difficulties (motion/mistimed bolus) & $H+$ & $+1++$ & $H$ & ++ \\
\hline Safety from radiation dose & ++ & ++ & + & $+1+$ \\
\hline
\end{tabular}

NCCT, noncontrast computed tomomgraphy; SCTA, single phase computed tomography angiography; mCTA, multiphase computed tomography angiography; CTP, computed tomography perfusion; mMRI, multimodal magnetic resonance imaging. 
shown in the ESCAPE trial.

\section{Noncontrast CT, multiphase CTA (1 step, 1 contrast)}

A combination of noncontrast $\mathrm{CT}$ and multiphase CTA is a refinement of the basic noncontrast $\mathrm{CT} /$ single phase CTA approach. The effectiveness of this protocol has been proven by the ESCAPE study. The study showed both a significant reduction in mortality and a shift toward better outcomes on modified Rankin Scale distribution despite no age limit and a longer time window (up to 12 hours) than other trials. This protocol does add about 20 seconds to the CT imaging protocol which increases radiation dose (approximately $0.5 \mathrm{mSV}$ per additional phase).

\section{Noncontrast CT, CTA, and CTP (1 step, 2 contrasts)}

CTP is a more sophisticated tool that was successfully implemented in two of the endovascular trials. It is capable of determining infarct core volumes with the use of automated computer software such as RAPID (iSchemaView). The EXTEND-IA trial reveals how robust CTP based selection can be with the largest numerical clinical benefit (33\% increase in modified Rankin Scale 0-2, not statistically different than the other trials though) from endovascular treatment amongst all the trials. ${ }^{12}$ This large treatment effect could alternatively be explained by the very high TICI $2 b / 3$ reperfusion rates achieved in the EXTEND-IA trial. A treatment cutoff of a small core volume will select a population with generally good prognosis whatever the therapy. The control arm of EXTEND-IA had a high proportion of good outcomes (40\%). ${ }^{12}$ Performing a CTP usually requires a second bolus of contrast after the CTA. Newer CT technology can perform a CTA and CTP with the same bolus of contrast although CTA neck imaging usually is not possible with such an approach. CTP adds a radiation dose of approximately $4 \mathrm{mSV}$ depending on the CT scanner involved. CTP also adds additional imaging time (2-3 minutes) and post-processing time (515 minutes). Standardization of CTP core volume definition varies by study and remains contentious. What is the best core volume measurement (Tmax, cerebral blood flow or cerebral blood volume) and what is the larger core volume that should exclude a patient from endovascular treatment? Eloquence of brain is also a critical factor in determining futility with endovascular treatment. Lesions in the right temporal lobe especially can be quite large but produce very few neurologic signs. Future trials in the later time windows such as unknown onset of stroke and wake-up stroke seem excellent populations to maximize the potential of CTP as a selection tool. ${ }^{35,36}$
Multimodal MRI (1 step, 1 contrast, and longer time)

Despite the simplicity of a noncontrast CT/single phase CTA or multiphase CTA approach there remains concerns that patients will be misclassified by such an approach. MRI via DWI is ideal for showing discrete lesions even in hyperacute period in the first 2 hours of stroke. ${ }^{53}$ RAPID (iSchemaView) has also been developed to shorten the process time for calculating mismatch volume or ratio in time. ${ }^{34}$ Both DEFUSE-2 and MR RESCUE have used this protocol. ${ }^{8,33}$ The previous MR RESCUE was a prospective randomized control trial that failed to document effectiveness of target mismatch. ${ }^{8}$ MR RESCUE had too long an onset to treatment time and too low a successful reperfusion rate, which limited interpretation of these negative results. One major issue limiting the use of the multimodal MRI protocol is added time required. Previous studies have demonstrated door to needle times that significantly longer than CT based protocols..$^{54,55} \mathrm{MRI}$ is likely to also significantly delay door to reperfusion times. A quick 6-minute protocol may be part of the answer; ${ }^{56}$ however, other delays prior to imaging such as screening and preparing the patient for the MRI environment add to the time delay. MRI as first line imaging should be reserved for centres who are extremely efficient in screening, prepping and performing acute stroke MRI. Close monitoring of door to reperfusion times are needed to justify this approach at such centres.

\section{Conclusions}

With the recently successful endovascular treatment trials it is time to evaluate the optimal imaging protocol for acute disabling stroke. Vascular imaging with CTA is essential to confirm an intracranial LAO and can also provide collateral flow information without further imaging (single phase CTA). Noncontrast brain CT scans can be used to estimate infarct volume with ASPECTS system as well as to exclude patients with hemorrhagic stroke. Additional imaging such as multiphase CTA or CTP can increase confidence estimating infarct core. Multiphase CTA has the advantage of simplicity since it only adds about 20 seconds to the single phase CTA protocol. CTP may not be necessary in the first six hours from onset where the efficacy of endovascular treatment is clearly proven. It may have a crucial role for more careful patient selection required for wakeup strokes and late window. Future trials are planning to use CTP for selection in these settings. Multimodal MRI is still an option at highly efficient centres as long as door to reperfusion times rival what can be now accomplished with NCCT/multiphase CTA protocols. 


\section{References}

1. The National Institute of Neurological Disorders and Stroke rt-PA Stroke Study Group. Tissue plasminogen activator for acute ischemic stroke. N Engl J Med 1995;333:1581-1587.

2. Hacke W, Kaste M, Bluhmki E, Brozman M, Davalos A, Guidetti D, et al. Thrombolysis with alteplase 3 to 4.5 hours after acute ischemic stroke. NEngl JMed 2008;359:1317-1329.

3. Bivard A, Lin L, Parsons MW. Review of Stroke Thrombolytics. J Stroke 2013;15:90-98.

4. Saqqur M, Uchino K, Demchuk AM, Molina CA, Garami Z, Calleja $S$, et al. Site of arterial occlusion identified by transcranial Doppler predicts the response to intravenous thrombolysis for stroke. Stroke 2007;38:948-954.

5. Bhatia R, Hill MD, Shobha N, Menon B, Bal S, Kochar P, et al. Low rates of acute recanalization with intravenous recombinant tissue plasminogen activator in ischemic stroke: real-world experience and a call for action. Stroke 2010;41:2254-2258.

6. Broderick JP, Palesch YY, Demchuk AM, Yeatts SD, Khatri P, Hill MD, et al. Endovascular therapy after intravenous t-PA versus t-PA alone for stroke. N Engl J Med 2013;368:893-903.

7. Ciccone A, Valvassori L, Nichelatti M, Sgoifo A, Ponzio M, Sterzi R, et al. Endovascular treatment for acute ischemic stroke. NEngl J Med 2013;368:904-913.

8. Kidwell CS, Jahan R, Saver JL. Endovascular treatment for acute ischemic stroke. N Engl J Med 2013;368:2434-2435.

9. Berkhemer OA, Fransen PS, Beumer D, van den Berg LA, Lingsma HF, Yoo AJ, et al. A Randomized Trial of Intraarterial Treatment for Acute Ischemic Stroke. N Engl J Med 2014;372:11-20.

10. Goyal M, Demchuk AM, Menon BK, Eesa M, Rempel JL, Thornton J, et al. Randomized assessment of rapid endovascular treatment of ischemic stroke. N Engl J Med 2015;372:1019-1030.

11. Saver JL, Goyal M, Bonafe A, Diener HC, Levy EI, Pereira VM, et al. Stent-Retriever Thrombectomy after Intravenous t-PA vs. t-PA Alone in Stroke. N Engl J Med 2015;372:2285-2295.

12. Campbell BC, Mitchell PJ, Kleinig TJ, Dewey HM, Churilov L, Yassi N, et al. Endovascular therapy for ischemic stroke with perfusion-imaging selection. N Engl J Med 2015;372:1009-1018.

13. Jovin TG, Chamorro A, Cobo E, de Miquel MA, Molina CA, Rovira A, et al. Thrombectomy within 8 Hours after Symptom Onset in Ischemic Stroke. N Engl J Med 2015;372:2296-2306.

14. Balasubramaian A, Mitchell P, Dowling R, Yan B. Evolution of Endovascular Therapy in Acute Stroke: implications of device development. J Stroke 2015;17:127-137.

15. Ding D. Endovascular Mechanical Thrombectomy for Acute Ischemic Stroke: A New Standard of Care. J Stroke 2015;17: 123-126.

16. Goyal M, Almekhlafi M, Menon B, Hill M, Fargen K, Parsons
$\mathrm{M}$, et al. Challenges of acute endovascular stroke trials. Stroke 2014;45:3116-3122.

17. Qureshi AI, Abd-Allah F, Aleu A, Connors JJ, Hanel RA, Hassan $\mathrm{AE}$, et al. Endovascular treatment for acute ischemic stroke patients: implications and interpretation of IMS III, MR RESCUE, and SYNTHESIS EXPANSION trials: a report from the Working Group of International Congress of Interventional Neurology. J Vasc Interv Neurol 2014;7:56-75.

18. Fransen PS, Beumer D, Berkhemer OA, van den Berg LA, Lingsma $H$, van der Lugt A, et al. MR CLEAN, a multicenter randomized clinical trial of endovascular treatment for acute ischemic stroke in the Netherlands: study protocol for a randomized controlled trial. Trials 2014;15:343.

19. Demchuk AM, Goyal M, Menon BK, Eesa M, Ryckborst KJ, Kamal N, et al. Endovascular treatment for Small Core and Anterior circulation Proximal occlusion with Emphasis on minimizing CT to recanalization times (ESCAPE) trial: methodology. Int J Stroke 2015;10:429-438.

20. Saver JL, Goyal M, Bonafe A, Diener HC, Levy EI, Pereira VM, et al. Solitaire with the Intention for Thrombectomy as Primary Endovascular Treatment for Acute Ischemic Stroke (SWIFT PRIME) trial: protocol for a randomized, controlled, multicenter study comparing the Solitaire revascularization device with IV tPA with IV tPA alone in acute ischemic stroke. Int $J$ Stroke 2015;10:439-448.

21. Campbell BC, Mitchell PJ, Yan B, Parsons MW, Christensen S, Churilov L, et al. A multicenter, randomized, controlled study to investigate EXtending the time for Thrombolysis in Emergency Neurological Deficits with Intra-Arterial therapy (EXTEND-IA). Int J Stroke 2014;9:126-132.

22. Molina CA, Chamorro A, Rovira A, de Miquel A, Serena J, Roman LS, et al. REVASCAT: a randomized trial of revascularization with SOLITAIRE FR(R) device vs. best medical therapy in the treatment of acute stroke due to anterior circulation large vessel occlusion presenting within eight-hours of symptom onset. Int J Stroke 2015;10:619-626.

23. Demchuk AM, Goyal M, Yeatts SD, Carrozzella J, Foster LD, Qazi E, et al. Recanalization and Clinical Outcome of Occlusion Sites at Baseline CT Angiography in the Interventional Management of Stroke III Trial. Radiology 2014:132649.

24. Demchuk AM, Menon BK, Eesa M, Willinsky RA, Montaner WJ, Frei DF, et al. Correlation between baseline imaging variables and clinica outcome: results from the ESCAPE trial. European Stroke Organization Conference 2015:410.

25. Barber PA, Demchuk AM, Zhang J, Buchan AM. Validity and reliability of a quantitative computed tomography score in predicting outcome of hyperacute stroke before thrombolytic therapy. ASPECTS Study Group. Alberta Stroke Programme 
Early CT Score. Lancet 2000;355:1670-1674.

26. Coutts SB, Demchuk AM, Barber PA, Hu WY, Simon JE, Buchan AM, et al. Interobserver variation of ASPECTS in real time. Stroke 2004;35:e103-105.

27. Gupta AC, Schaefer PW, Chaudhry ZA, Leslie-Mazwi TM, Chandra RV, Gonzalez RG, et al. Interobserver reliability of baseline noncontrast CT Alberta Stroke Program Early CT Score for intra-arterial stroke treatment selection. AJNR Am J Neuroradiol 2012;33:1046-1049.

28. Bal S, Bhatia R, Menon BK, Shobha N, Puetz V, Dzialowski I, et al. Time dependence of reliability of noncontrast computed tomography in comparison to computed tomography angiography source image in acute ischemic stroke. Int J Stroke 2015; 10:55-60.

29. Demchuk AM, Hill MD, Barber PA, Silver B, Patel SC, Levine SR. Importance of early ischemic computed tomography changes using ASPECTS in NINDS rtPA Stroke Study. Stroke 2005; 36:2110-2115.

30. Yoo AJ, Zaidat OO, Chaudhry ZA, Berkhemer OA, Gonzalez RG, Goyal M, et al. Impact of pretreatment noncontrast CT Alberta Stroke Program Early CT Score on clinical outcome after intra-arterial stroke therapy. Stroke 2014;45:746-751.

31. Hill MD, Demchuk AM, Goyal M, Jovin TG, Foster LD, Tomsick TA, et al. Alberta Stroke Program early computed tomography score to select patients for endovascular treatment: Interventional Management of Stroke (IMS)-III Trial. Stroke 2014; 45:444-449.

32. McTaggart RA, Jovin TG, Lansberg MG, Mlynash M, Jayaraman MV, Choudhri OA, et al. Alberta stroke program early computed tomographic scoring performance in a series of patients undergoing computed tomography and MRI: reader agreement, modality agreement, and outcome prediction. Stroke 2015;46:407-412.

33. Lansberg MG, Straka M, Kemp S, Mlynash M, Wechsler LR, Jovin TG, et al. MRI profile and response to endovascular reperfusion after stroke (DEFUSE 2): a prospective cohort study. Lancet Neurol 2012;11:860-867.

34. Straka M, Albers GW, Bammer R. Real-time diffusion-perfusion mismatch analysis in acute stroke. J Magn Reson Imaging 2010;32:1024-1037.

35. Hassan AE, Zacharatos H, Rodriguez GJ, Vazquez G, Miley JT, Tummala RP, et al. A comparison of Computed Tomography perfusion-guided and time-guided endovascular treatments for patients with acute ischemic stroke. Stroke 2010;41:16731678.

36. Turk AS, Nyberg EM, Chaudry MI, Turner RD, Magarik JA, Nicholas JS, et al. Utilization of CT perfusion patient selection for mechanical thrombectomy irrespective of time: a compari- son of functional outcomes and complications. J Neurointerv Surg 2013;5:518-522.

37. Lin L, Bivard A, Parsons MW. Perfusion Patterns of Ischemic Stroke on Computed Tomography Perfusion. J Stroke 2013; 15:164-173.

38. Shuaib A, Butcher K, Mohammad AA, Saqqur M, Liebeskind DS. Collateral blood vessels in acute ischaemic stroke: a potential therapeutic target. Lancet Neurol 2011;10:909-921.

39. Menon BK, Smith EE, Modi J, Patel SK, Bhatia R, Watson TW, et al. Regional leptomeningeal score on CT angiography predicts clinical and imaging outcomes in patients with acute anterior circulation occlusions. AJNR Am J Neuroradiol 2011; 32:1640-1645.

40. Kim JJ, Fischbein NJ, Lu Y, Pham D, Dillon WP. Regional angiographic grading system for collateral flow: correlation with cerebral infarction in patients with middle cerebral artery occlusion. Stroke 2004;35:1340-1344.

41. Christoforidis GA, Mohammad Y, Kehagias D, Avutu B, Slivka AP. Angiographic assessment of pial collaterals as a prognostic indicator following intra-arterial thrombolysis for acute ischemic stroke. AJNR Am J Neuroradiol 2005;26:1789-1797.

42. Qureshi AI. New grading system for angiographic evaluation of arterial occlusions and recanalization response to intra-arterial thrombolysis in acute ischemic stroke. Neurosurgery 2002; 50:1405-1414; discussion 1414-1405.

43. Christoforidis GA, Karakasis C, Mohammad Y, Caragine LP, Yang M, Slivka AP. Predictors of hemorrhage following intra-arterial thrombolysis for acute ischemic stroke: the role of pial collateral formation. AJNR Am J Neuroradiol 2009;30:165-170.

44. Bang OY, Saver JL, Kim SJ, Kim GM, Chung CS, Ovbiagele B, et al. Collateral flow averts hemorrhagic transformation after endovascular therapy for acute ischemic stroke. Stroke 2011; 42:2235-2239.

45. Miteff F, Levi CR, Bateman GA, Spratt N, McElduff P, Parsons MW. The independent predictive utility of computed tomography angiographic collateral status in acute ischaemic stroke Brain 2009; 132:2231-2238.

46. Maas MB, Lev MH, Ay H, Singhal AB, Greer DM, Smith WS, et al. Collateral vessels on $\mathrm{CT}$ angiography predict outcome in acute ischemic stroke. Stroke 2009;40:3001-3005.

47. Tan IY, Demchuk AM, Hopyan J, Zhang L, Gladstone D, Wong $\mathrm{K}$, et al. CT angiography clot burden score and collateral score: correlation with clinical and radiologic outcomes in acute middle cerebral artery infarct. AJNR Am J Neuroradiol 2009;30: 525-531.

48. Schramm P, Schellinger PD, Fiebach JB, Heiland S, Jansen O, Knauth $\mathrm{M}$, et al. Comparison of CT and CT angiography source images with diffusion-weighted imaging in patients 
with acute stroke within 6 hours after onset. Stroke 2002;33:

2426-2432.

49. Rosenthal ES, Schwamm LH, Roccatagliata L, Coutts SB, Demchuk AM, Schaefer PW, et al. Role of recanalization in acute stroke outcome: rationale for a CT angiogram-based "benefit of recanalization" model. AJNR Am J Neuroradiol 2008;29:1471-1475.

50. Menon BK, Qazi E, Nambiar V, Foster LD, Yeatts SD, Liebeskind D, et al. Differential Effect of Baseline Computed Tomographic Angiography Collaterals on Clinical Outcome in Patients Enrolled in the Interventional Management of Stroke III Trial. Stroke 2015;46:1239-1244.

51. Smit EJ, Vonken EJ, van Seeters T, Dankbaar JW, van der Schaaf IC, Kappelle LJ, et al. Timing-invariant imaging of collateral vessels in acute ischemic stroke. Stroke 2013;44:2194-2199.

52. Menon BK, d'Esterre CD, Qazi EM, Almekhlafi M, Hahn L, Demchuk AM, et al. Multiphase CT Angiography: a new tool for the imaging triage of patients with acute ischemic stroke. Radiology 2015;275:510-520.

53. Kim BJ, Kang HG, Kim H-J, Ahn SH, Kim NY, Warach S, et al. Magnetic Resonance Imaging in Acute Ischemic Stroke Treatment. J Stroke 2014;16:131-145.

54. Yoo SH, Kwon SU, Lee DH, Kim SJ, Kim JS, Kang DW. Comparison between MRI screening and CT-plus-MRI screening for thrombolysis within $3 \mathrm{~h}$ of ischemic stroke. J Neurol Sci 2010;294:119-123.

55. Kang DW, Chalela JA, Dunn W, Warach S. MRI screening before standard tissue plasminogen activator therapy is feasible and safe. Stroke 2005;36:1939-1943.

56. Nael K, Khan R, Choudhary G, Meshksar A, Villablanca P, Tay $\mathrm{J}$, et al. Six-minute magnetic resonance imaging protocol for evaluation of acute ischemic stroke: pushing the boundaries. Stroke 2014;45:1985-1991. 